\title{
Erratum to: Enhancing the catalytic potential of nitrilase from Pseudomonas putida for stereoselective nitrile hydrolysis
}

\author{
Anirban Banerjee $^{1} \cdot$ Praveen Kaul $^{1}$ - U. C. Banerjee ${ }^{1}$
}

Published online: 8 July 2017

(C) Springer-Verlag GmbH Germany 2017

\section{Erratum to: Appl Microbiol Biotechnol \\ DOI 10.1007/s00253-005-0255-8}

In the original version of our paper entitled "Enhancing the catalytic potential of nitrilase from Pseudomonas putida for stereoselective nitrile hydrolysis" published in "Applied Microbiology and Biotechnology" (2006) 72: $77-87$, some references to already published articles were inadvertently left out.

1. Figure 1 has previously been published in the paper by Renu Singh, Anirban Banerjee, Praveen Kaul, Brajesh Barse, U. C. Banerjee, "Release of an enantioselective nitrilase from Alcaligenes faecalis MTCC 126: a comparative study", Bioprocess and Biosystems Engineering, October 2005, Volume 27, Issue 6, pp: 415-424.

2. In the paragraph on "Kinetic Model in the immobilized cell systems" on page 83-84 Sections (1) and (2) describ-

ing the equation for mass transfer model have been taken from a paper by Chung, Tsuey-Ping, Hsiu-Ya Tseng, and Ruey-Shin Juang. "Mass transfer effect and intermediate detection for phenol degradation in immobilized Pseudomonas putida systems". Process Biochemistry 38.10 (2003): 1497-1507." License for the same has been acquired [License Number 4114020240483] but the reference was missing.

The final list of missing references is as follows:

Chung, Tsuey-Ping, Hsiu-Ya Tseng, and Ruey-Shin Juang. "Mass transfer effect and intermediate detection for phenol degradation in immobilized Pseudomonas putida systems." Process Biochemistry 38.10 (2003): 1497-1507.

Singh, Renu, et al. "Release of an enantioselective nitrilase from Alcaligenes faecalis MTCC 126: a comparative study." Bioprocess and Biosystems Engineering 27.6 (2005): 415-424.

The online version of the original article can be found at http://dx.doi. org/10.1007/s00253-005-0255-8

U. C. Banerjee

ucbanerjee@niper.ac.in

Department of Pharmaceutical Technology (Biotechnology), National Institute of Pharmaceutical Education and Research Sector-67, SAS Nagar, Punjab 160 062, India 